\title{
Multi-agent Kalman Consensus with Relative Uncertainty
}

\author{
Wei Ren, Randal W. Beard, Derek B. Kingston
}

\begin{abstract}
In this paper, we propose discrete-time and continuous-time consensus update schemes motivated by the discrete-time and continuous-time Kalman filters. With certainty information encoded into each agent, the proposed consensus schemes explicitly account for relative confidence / reliability of information states from each agent in the team. We show mild sufficient conditions under which consensus can be achieved using the proposed consensus schemes in the presence of switching interaction topologies.
\end{abstract}

\section{INTRODUCTION}

In recent years, there has been significant interest and research activity in the area of coordinated and cooperative control. Much of this work assumes the availability of global team knowledge, and/or the ability to plan group actions in a centralized manner. Centralized coordination techniques are suitable if each member of the team has the ability to communicate to a centralized location or if the team is able to share information via a static fully connected network. On the other hand, real-world communication topologies are usually not fully connected. In many cases they depend on the relative position of the vehicles and on other environmental factors and are therefore dynamically changing in time. In addition, wireless communication channels are subject to multi-path, fading and drop-out. Therefore, cooperative control in the presence of realworld communication constraints, becomes a significant challenge.

In a recent article we argued that "shared information is a necessary condition for cooperation" [1]. Shared information may take the form of common objectives, common control algorithms, relative position information, or a world map. If this assertion is true, then information exchange becomes a central issue in cooperative control. In this article, we will refer to the information that is necessary for coordination as the coordination information or coordination variable [2]. In the presence of an unreliable, dynamically changing communication topology, it is not possible for all of the vehicles to have access to identical coordination information. Suppose that a particular cooperation strategy has been devised and shown to work if the team has global access to the coordination information. Cooperation will occur if each member on the team has access to the same information.

As an example, consider the meet-for-dinner problem introduced in [1]. In this problem, a group of friends decide to meet for dinner at a particular restaurant but fail to specify a precise time to meet. On the afternoon of the dinner appointment, each individual realizes that they are uncertain about the time that the group will meet for dinner. A centralized solution to this problem is for the group to have a conference call, to poll each individual regarding

W. Ren is with the Space Systems Laboratory at the University of Maryland. weirenass 1 . umd. edu

R. W. Beard and D. B. Kingston are with the Department of Electrical and Computer Engineering at Brigham Young University. their preferred time for dinner, and to average the answers to arrive at a time that the group will meet for dinner. However, this centralized solution requires that a conference line is available, and that the time of the conference call is known to the group. Since, whatever algorithm was used to convey the time of the conference call to the group, could also have been used to convey the time to meet for dinner, the central problem remains.

The information variable in this example is the time that the group will meet for dinner. The particular time is not what is important, but rather that each individual in the group has a consistent understanding of that information. A decentralized solution to the problem would be for each individual to call, one at a time, a subset of the group. Given his current estimate of the meeting time, the individual might update his estimate of the meeting time to be a weighted average of his current meeting time and that of the person with whom he is conversing. The question (which will be answered in this paper) is under what conditions this strategy will enable the entire team to converge to a consistent meeting time.

Convergence to a consistent view of the coordination variable in the presence of an unreliable, dynamically changing communication topology is called the consensus problem. Consensus problems have recently been addressed in [3], [4], [5], [6], [7], [1], [8], [9], to name a few. Previous consensus seeking results reported in the literature do not explicitly account for agent confidence in their instantiation of the coordination variable. Most results assume that each individual in the group has identical confidence in their instantiation of the coordination variable. However, there are many cases where some individuals on the team will have access to better information than others. In cases like these the consensus algorithm needs to be biased to favor vehicles with better information. For example, if a team of UAVs is tasked with tracking the location of a group of ground vehicles, the quality of information will be proportional to the relative sensing distance. UAVs that have recently flown close to a ground vehicle should be considered more reliable than those that are sensing from a greater distance, or whose information is old. As another example, in the meet-for-dinner problem described above, if one individual is considered more reliable than the others, his/her information should be weighted more heavily when making the team decision.

The primary contribution of this paper is to derive continuous-time and discrete-time consensus strategies, based on a Kalman-filter structure, that asymptotically achieves consensus in the presence of an unreliable, dynamically changing communication topology, giving proper weight to individuals with greater certainty in their coordination variable. 


\section{KALMAN-Filter APPROACH TO Multi-AGENT CONSENSUS}

The Kalman filter is used extensively to estimate a system's current state from imprecise measurement data [10], [11], [12]. It is well-known that the Kalman filter is an optimal estimator in the case of Gaussian statistics and that it is the best linear estimator in the case of other statistics [13]. Motivated by the Kalman filter scheme, we treat the final consensus value as the system state, which is unknown a priori but is the final equilibrium state that each agent in the group is expected to achieve. In the consensus problem, each agent has an estimate of the final consensus value. Communication from other agents regarding their estimate of the final consensus value will be regarded as measurement data. In this sense, each agent in the group performs its own estimate of the final consensus value based on the information available to it. Our goal is to guarantee that the information state of each agent achieves the final consensus value. In other words, the objective is to minimize the mean squared error between each agent's estimate of the coordination variable and the final consensus value. The error covariance matrix is interpreted as the confidence that each agents has in its current estimate of the coordination variable, where large covariance indicates low confidence, and small covariance indicates a high degree of confidence.

\section{A. Continuous-time Consensus}

Table II-A: Continuous-time Kalman filter [14].

$$
\begin{aligned}
& \text { System model and measurement model: } \\
& \dot{x}=A x+B u+G w, \quad z=H x+v \\
& x(0) \sim\left(\bar{x}_{0}, P_{0}\right), w \sim(0, Q), v \sim(0, R)
\end{aligned}
$$

Assumptions: $\{w(t)\}$ and $\{v(t)\}$ are white noise processes

uncorrelated with $x(0)$ and with each other. $R$ is positive definite.

Initialization: $P(0)=P_{0}, \hat{x}(0)=\bar{x}_{0}$

Error covariance update:

$$
\dot{P}=A P+P A^{T}+G Q G^{T}-P H^{T} R^{-1} H P
$$

Kalman gain: $K=P H^{T} R^{-1}$

Estimate update: $\dot{\hat{x}}=A \hat{x}+B u+K(z-H \hat{x})$

The standard continuous-time Kalman filter is summarized in Table II-A [14]. The objective of this section is to show how the Kalman filter equations can be used to derive a decentralized information consensus scheme.

Let $\xi^{*} \in \mathbb{R}^{m}$ be the a priori unknown information state over which the team is to form consensus. In other words, each information state $\xi_{i}$ will converge to the consensus value $\xi^{*}$ as $t \rightarrow \infty$. Note that the consensus value will depend not only on interaction topologies but on the weighting factors in the update schemes. In this paper we will assume that the consensus state is a constant, which implies that the system dynamics are given by $\dot{\xi}^{*}=w$, where, with reference to Table II-A, $A=0, B=0$, and $G=I_{m}$. The case of more complicated system dynamics is more involved and will be a topic of future research. In the following, we assume that $Q(t)$ is positive definite and uniformly lower and upper bounded.

Treat the $i^{\text {th }}$ information state $\xi_{i}$ as the $i^{\text {th }}$ agent's estimate of $\xi^{*}$ and suppose that the $j^{\text {th }}$ agent communicates $\xi_{j}$ to the $i^{\text {th }}$ agent with transmission, or communication noise $\nu_{i j}$. Also, let $g_{i j}(t)$ be a time-varying boolean variable that indicates the presence of an open communication channel from agent $j$ to agent $i$ at time $t$, i.e., $g_{i j}(t)=1$ if information is communicated from $j$ to $i$ at time $t$ and zero otherwise. Note that $g_{i i}(t) \triangleq 1$. Using these definitions, it is clear that the measurement model of the $i^{\text {th }}$ agent can be given by

$$
z_{i}=\left(\begin{array}{c}
g_{i 1}\left(\xi_{1}+\nu_{i 1}\right) \\
\vdots \\
g_{i n}\left(\xi_{n}+\nu_{i n}\right)
\end{array}\right)=\left(\begin{array}{c}
g_{i 1} I \\
\vdots \\
g_{i n} I
\end{array}\right) \xi^{*}+\left(\begin{array}{c}
g_{i 1}\left(\xi_{1}-\xi^{*}+\nu_{i 1}\right) \\
\vdots \\
g_{i n}\left(\xi_{n}-\xi^{*}+\nu_{i n}\right)
\end{array}\right),
$$

where, with reference to Table II-A, $H_{i}^{T}=\left(g_{i 1} I \ldots g_{i n} I\right)$ and $v_{i}=\left(\begin{array}{c}g_{i 1}\left(\xi_{1}-\xi^{*}+\nu_{i 1}\right) \\ \vdots \\ g_{i n}\left(\xi_{n}-\xi^{*}+\nu_{i n}\right)\end{array}\right)$. If we define $P_{i} \triangleq$ $E\left\{\left(\xi_{i}-\xi^{*}\right)\left(\xi_{i}-\xi^{*}\right)^{T}\right\}$ and assume that $E\left\{\left(\xi_{i}-\xi^{*}\right)\left(\xi_{j}-\right.\right.$ $\left.\left.\xi^{*}\right)^{T}\right\}=0$, where $i \neq j$, then

$$
R_{i} \triangleq E\left\{v_{i} v_{i}^{T}\right\}=\left(\begin{array}{ccc}
g_{i 1}\left(P_{1}+\Omega_{i 1}\right) & \ldots & 0 \\
\vdots & \ddots & \vdots \\
0 & \ldots & g_{i n}\left(P_{n}+\Omega_{i n}\right)
\end{array}\right),
$$

where $\Omega_{i j} \triangleq E\left\{\nu_{i j} \nu_{i j}^{T}\right\}$ is assumed to be upper bounded. Therefore, the error covariance update in Table II-A becomes

$$
\dot{P}_{i}=-P_{i} H_{i}^{T} R_{i}^{-1} H_{i} P_{i}+Q=-P_{i}\left[\sum_{j=1}^{n} g_{i j}\left(P_{j}+\Omega_{i j}\right)^{-1}\right] P_{i}+Q .
$$

Similarly, the Kalman gain is given by

$K_{i}=P_{i} H_{i}^{T} R_{i}^{-1}=\left(g_{i 1} P_{i}\left(P_{1}+\Omega_{i 1}\right)^{-1} \cdots g_{\text {in }} P_{i}\left(P_{n}+\Omega_{i n}\right)^{-1}\right)$,

and the estimate update is given by

$$
\begin{aligned}
\dot{\xi}_{i} & =K_{i}\left(z_{i}-H_{i} \xi_{i}\right) \\
& =K_{i}\left[\left(\begin{array}{c}
g_{i 1}\left(\xi_{1}+\nu_{i 1}\right) \\
\vdots \\
g_{i n}\left(\xi_{n}+\nu_{i n}\right)
\end{array}\right)-\left(\begin{array}{c}
g_{i 1} I_{m} \\
\vdots \\
g_{i n} I_{m}
\end{array}\right) \xi_{i}\right] \\
& =\sum_{j=1}^{n} K_{i j} g_{i j}\left(\xi_{j}-\xi_{i}+\nu_{i j}\right),
\end{aligned}
$$

where $K_{i}=\left[K_{i 1}, K_{i 2}, \cdots, K_{i n}\right]$.

Summarizing, we have the following Kalman consensus scheme for the $i^{\text {th }}$ agent:

$$
\begin{aligned}
\dot{P}_{i} & =-P_{i}\left[\sum_{j} g_{i j}(t)\left(P_{j}+\Omega_{i j}\right)^{-1}\right] P_{i}+Q \\
K_{i j} & =P_{i}\left(P_{j}+\Omega_{i j}\right)^{-1} \\
\dot{\xi}_{i} & =\sum_{j=1}^{n} g_{i j}(t) K_{i j}\left(\left(\xi_{j}+\nu_{i j}\right)-\xi_{i}\right) .
\end{aligned}
$$

Note that Eq. (1) indicates that the certainty of information increases with communication but decreases with the size of the process noise. In addition, the rate of increase in certainty for the $i^{\text {th }}$ agent is inversely proportional to the certainty of the $j^{\text {th }}$ agent and the communication noise. Note also that the Kalman gain $K_{i j}$ is reduced if either the communication noise is large, or if the certainty of the $j^{\text {th }}$ agent is small (hence $P_{j}$ large). Note that Eq. (3) is similar to the continuous-time consensus schemes proposed in [4], [5], [1] except that the consensus gain $K_{i j}$ is time-varying in (3), and the communication noise is explicitly included. 
Table II-B: Discrete-time Kalman filter [14].

System model and measurement model:

$x[k+1]=A[k] x[k]+B[k] u[k]+G[k] w[k]$,

$z[k]=H[k] x[k]+v[k]$

$x(0) \sim\left(\bar{x}_{0}, P_{x_{0}}\right), w[k] \sim(0, Q[k]), v[k] \sim(0, R[k])$

Assumptions: $\{w[k]\}$ and $\{v[k]\}$ are white noise processes

uncorrelated with $x_{0}$ and with each other. $R[k]$ is positive definite.

Initialization: $P[0]=P_{x_{0}}, \hat{x}_{0}=\bar{x}_{0}$

Time update: (effect of system dynamics)

error covariance: $P[k+1]^{-}=A[k] P[k] A[k]^{T}+G[k] Q[k] G[k]^{T}$

estimte: $\hat{x}[k+1]^{-}=A[k] \hat{x}[k]+B[k] u[k]$

Measurement update: (effect of measurement $z[k]$ )

error covariance: $P[k+1]=\left[\left(P[k+1]^{-}\right)^{-1}\right.$

$\left.+H[k+1]^{T} R[k+1]^{-1} H[k+1]\right]^{-1}$

estimate: $\hat{x}[k+1]=\hat{x}[k+1]-$

$+P[k+1] H[k+1]^{T} R[k+1]^{-1}\left(z[k+1]-H[k+1] \hat{x}[k+1]^{-}\right)$

\section{B. Discrete-time Consensus}

The standard discrete-time Kalman filter is summarized in Table II-B [14]. Again assuming that $\xi^{*}$ is constant we get $\xi^{*}[k+1]=\xi^{*}[k]+w[k]$, where, with reference to Table II-A, $A[k]=I, B[k]=0$, and $G[k]=I_{m}$.

Again letting $\nu_{i j}[k]$ represent the communication noise, the measurement model for the $i^{\text {th }}$ agent can be given by

$$
\begin{aligned}
z_{i}[k] & =\left(\begin{array}{c}
g_{i 1}[k]\left(\xi_{1}[k]+\nu_{i 1}[k]\right) \\
\vdots \\
g_{i n}[k]\left(\xi_{n}[k]+\nu_{i n}[k]\right)
\end{array}\right) \\
& =\left(\begin{array}{c}
g_{i 1}[k] I \\
\vdots \\
g_{i n}[k] I
\end{array}\right) \xi^{*}[k]+\left(\begin{array}{c}
g_{i 1}[k]\left(\xi_{1}[k]-\xi^{*}[k]+\nu_{i 1}[k]\right) \\
\vdots \\
g_{i n}[k]\left(\xi_{n}[k]-\xi^{*}[k]+\nu_{i n}[k]\right)
\end{array}\right),
\end{aligned}
$$

where, with reference to Table II-A, $H_{i}^{T}[k]=\left(g_{i 1}[k] I \ldots g_{i n}[k] I\right)$ and $v_{i}=$ $\left(\begin{array}{c}g_{i 1}[k]\left(\xi_{1}[k]-\xi^{*}[k]+\nu_{i 1}[k]\right) \\ \vdots\end{array}\right) . \quad$ If we define $P_{i}[k] \triangleq E\left\{\left(\xi_{i}[k]-\xi^{*}[k]\right)\left(\xi_{i}[k]-\xi^{*}[k]\right)^{T}\right\}$ and assume that $E\left\{\left(\xi_{i}[k]-\xi^{*}[k]\right)\left(\xi_{j}[k]-\xi^{*}[k]\right)^{T}\right\}=0$, where $i \neq j$, then

$$
\begin{aligned}
R_{i}[k] & \triangleq E\left\{v_{i}[k] v_{i}[k]^{T}\right\} \\
& =\left(\begin{array}{ccc}
g_{i 1}[k]\left(P_{1}[k]+\Omega_{i 1}[k]\right) & \ldots & 0 \\
\vdots & \ddots & \vdots \\
0 & \ldots & g_{i n}[k]\left(P_{n}[k]+\Omega_{i n}[k]\right)
\end{array}\right),
\end{aligned}
$$

where $\Omega_{i j}[k] \triangleq E\left\{\nu_{i j}[k] \nu_{i j}[k]^{T}\right\}$.

Therefore, the error covariance update in Table II-B becomes $P_{i}^{-}[k+1]=P_{i}[k]+Q[k], \quad \xi_{i}^{-}[k+1]=\xi_{i}[k]$.

The measurement update is given by

$$
\begin{aligned}
P_{i} & {[k+1] } \\
= & {\left[\left(P_{i}^{-}[k+1]\right)^{-1}+H_{i}^{T}[k+1] R_{i}^{-1}[k][k+1] H_{i}[k+1]\right]^{-1} } \\
= & {\left[\left(P_{i}[k]+Q[k]\right)^{-1}+\sum_{j=1}^{n} g_{i j}[k]\left(P_{j}[k]+\Omega_{i j}[k]\right)^{-1}\right]^{-1} } \\
\xi_{i}[k+1] & \xi^{-}[k+1]+P_{i}[k+1] H_{i}^{T}[k+1] R_{i}^{-1}[k+1] . \\
& \left(z_{i}[k+1]-H_{i}[k+1] \xi^{-}[k+1]\right) \\
= & \xi_{i}[k]+P_{i}[k+1] . \\
& \sum_{j=1}^{n}\left[g_{i j}[k]\left(P_{j}[k]+\Omega_{i j}[k]\right)^{-1}\left(\left(\xi_{j}[k]+\nu_{i j}[k+1]\right)-\xi_{i}[k]\right)\right] .
\end{aligned}
$$

Summarizing, we have the following discrete-time Kalman consensus scheme for the $i^{\text {th }}$ agent:

$$
\begin{aligned}
& P_{i}[k+1] \\
& =\left[\left(P_{i}[k]+Q[k]\right)^{-1}+\sum_{j=1}^{n} g_{i j}[k]\left(P_{j}[k]+\Omega_{i j}[k]\right)^{-1}\right]^{-1} \\
& \xi_{i}[k+1] \\
& =\xi_{i}[k]+P_{i}[k+1] . \\
& \left.\quad \sum_{j=1}^{n}\left[g_{i j}[k]\left(P_{j}[k]+\Omega_{i j}[k]\right)^{-1}\left(\xi_{j}[k]+\nu_{i j}[k+1]\right)-\xi_{i}[k]\right)\right],
\end{aligned}
$$

\section{Meet for Dinner Example}

To illustrate, consider the meet-for-dinner problem discussed in the introduction. Suppose that there are $n=10$ agents who communicate with exactly one other individual, chosen randomly from the group, for a random length of time. After the communication has expired, the process is repeated. Figure 1 shows the state and variance plots under
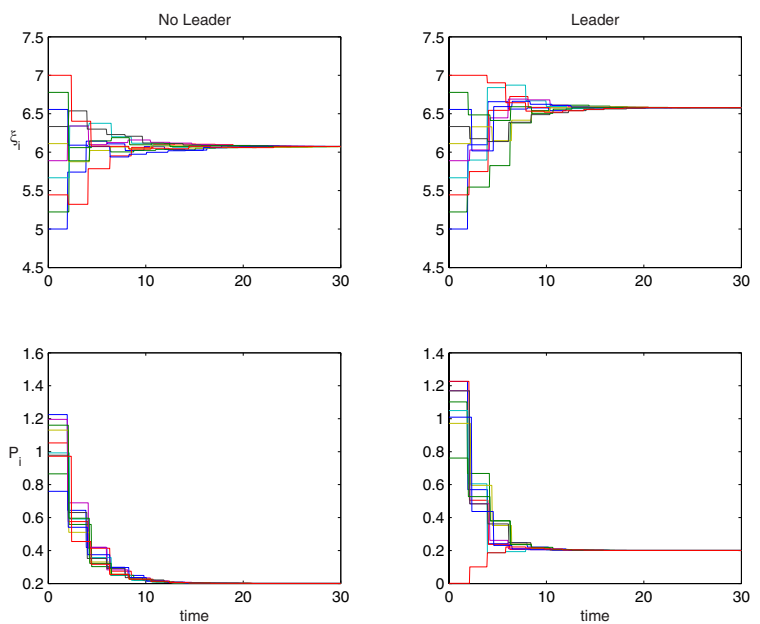

Fig. 1. Discrete time Meet-for-Dinner simulation. The subplot in the upper left shows the evolution of the coordination variable assuming that all agents begin with equally confident covariance. The subplot in the lower left shows the associated covariance. The subplots on the right show identical data where the agent with initial time $\xi_{i}=7$ has an initial covariance of $P_{i}=0.001$.

the discrete-time Kalman consensus scheme (4)-(5) where the initial state is uniformly assigned. The subplots on the left show the arrival times and variance when the initial variances are uniformly assigned. The subplots on the right show the arrival times and variances when the variance of the agent with initial arrival time $\xi_{i}=7$ is given an initial variance of $P_{i}=0.001$, which is significantly lower than the other agents. Note that in this case, the final consensus value is influenced to a greater degree by this agent. The simulation uses the values $\Omega_{i j}=0.1$ and $Q=0.1$.

\section{Convergence Results}

The objective of this section is to state some technical properties of the algorithms given in Eqs. (1)-(3) and Eqs. (4)-(5). For notational simplicity, we will focus on the case where each information state $\xi^{*}$ is a scalar. The vector case reduces to the scalar case if $P_{i 0}$ is a diagonal matrix. 
The general case where $P_{i 0}$ is non-diagonal is currently a topic of research.

\section{A. Preliminaries}

Let $\mathcal{A}=\left\{A_{i} \mid i \in \mathcal{I}\right\}$, where $\mathcal{I}=\{1,2, \cdots, n\}$, be a set of $n$ agents among whom consensus is desired. A directed graph $\mathcal{G}$ will be used to model the interaction topology among these agents. In $\mathcal{G}$, the $i^{\text {th }}$ vertex represents the $i^{\text {th }}$ agent $A_{i}$ and a directed edge from $A_{i}$ to $A_{j}$ denoted as $\left(A_{i}, A_{j}\right)$ represents a unidirectional information exchange from $A_{i}$ to $A_{j}$, that is, agent $j$ receives information from agent $i,(i, j) \in \mathcal{I}$. If the information flows from agent $i$ to agent $j$, agent $i$ is called the parent of $j$, and agent $j$ is called the child of $i$. A directed path in graph $\mathcal{G}$ is a sequence of edges $\left(A_{i_{1}}, A_{i_{2}}\right),\left(A_{i_{2}}, A_{i_{3}}\right),\left(A_{i_{3}}, A_{i_{4}}\right), \cdots$ in that graph. Graph $\mathcal{G}$ is called strongly connected if there is a directed path from $A_{i}$ to $A_{j}$ and $A_{j}$ to $A_{i}$ between any pair of distinct vertices $A_{i}$ and $A_{j}, \forall(i, j) \in \mathcal{I}$. A directed tree is a directed graph, where every node, except the root, has exactly one parent. A spanning tree of a directed graph is a directed tree formed by graph edges that connect all the vertices of the graph [15]. We say that a directed graph has a spanning tree if there exists a spanning tree that is a subset of the directed graph. Fig. 2 shows a directed graph with more than one possible spanning trees. The double arrows denote one possible spanning tree with $A_{5}$ as the parent. Spanning trees with $A_{1}$ and $A_{4}$ as the parent, are also possible. As a comparison, Figs. 3 shows two cases where the graph does not have a spanning tree.

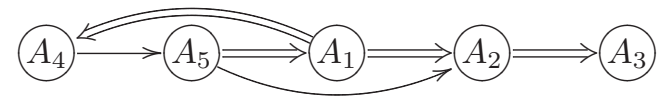

Fig. 2. A directed graph that has more than one possible spanning trees, but is not strongly connected. One possible spanning tree is denoted with double arrows.
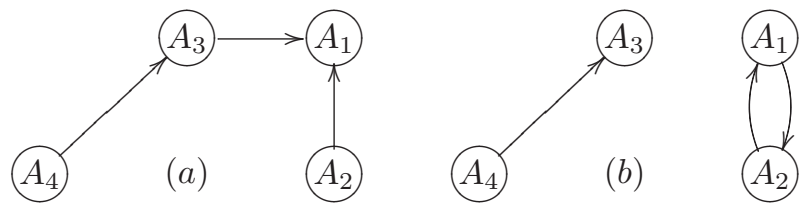

Fig. 3. (a) A directed graph that has two leaders, and hence does not contain a spanning tree. (b) A directed graph that has two isolated groups, and hence does not contain a spanning tree.

The interaction topology may change dynamically. Let $\overline{\mathcal{G}}=\left\{\mathcal{G}_{1}, \mathcal{G}_{2}, \cdots, \mathcal{G}_{M}\right\}$ denote the set of all possible directed interaction graphs defined for $\mathcal{A}$. It is obvious that $\overline{\mathcal{G}}$ has a finite number of elements and that $\mathcal{G}(t) \in \overline{\mathcal{G}}$. The union of a set of directed graphs $\left\{\mathcal{G}_{i_{1}}, \mathcal{G}_{i_{2}}, \cdots, \mathcal{G}_{i_{m}}\right\} \subset \overline{\mathcal{G}}$ is a directed graph with vertices given by $A_{i}, i \in \mathcal{I}$ and edge set given by the union of the edge sets of $\mathcal{G}_{i_{j}}, j=1, \cdots, m$. We will assume throughout the paper that the interaction topology does not switch infinitely fast.

Let $M_{n}(\mathbb{R})$ represent the set of all $n \times n$ real matrices. Given a matrix $A=\left[a_{i j}\right] \in M_{n}(\mathbb{R})$, the directed graph of $A$, denoted by $\Gamma(A)$, is the directed graph on $n$ vertices $V_{i}$, $i \in \mathcal{I}$, such that there is a directed edge in $\Gamma(A)$ from $V_{j}$ to $V_{i}$ if and only if $a_{i j} \neq 0$ [16].
A matrix $A=\left[a_{i j}\right] \in M_{n}(\mathbb{R})$ is nonnegative, denoted as $A \geq 0$, if all its entries are nonnegative. Furthermore, if all its row sums are $+1, A$ is said to be a (row) stochastic matrix [16]. A stochastic matrix $P$ is called indecomposable and aperiodic (SIA) if $\lim _{n \rightarrow \infty} P^{n}=\mathbf{1} y^{T}$, where $y$ is a column vector, and 1 denotes an $n \times 1$ column vector with all the entries equal to 1 [17]. For nonnegative matrices, $A \geq B$ implies that $A-B$ is a nonnegative matrix. It is easy to verify that if $A \geq \rho B$, for some $\rho>0$, then the directed graph of $B$ is a subset of the directed graph of $A$.

Two $n \times n$ nonnegative matrices are said to be of the same type if their zero elements are in the same locations [17]. We will use the notation $P \sim Q$ to denote that $P$ and $Q$ are of the same type.

Lemma 3.1: Given $n \times n$ nonnegative matrices $P, Q, R$, and $S$, if $P \sim R$ and $Q \sim S$, then $(P+Q) \sim(R+S)$ and $P Q \sim R S$. Moreover, if a time-varying nonnegative matrix $M(t)$ with continuous entries is of a fixed type for $t \in\left[t_{1}, t_{2}\right]$, where $t_{1}<t_{2}$, then $M(t) \sim \int_{t_{1}}^{t_{2}} M(t) d t$.

Proof: Trivial.

Let $\xi_{i} \in \mathbb{R}, i \in \mathcal{I}$, represent the $i^{\text {th }}$ deterministic information state associated with the $i^{\text {th }}$ agent. The set of agents $\mathcal{A}$ is said to achieve consensus asymptotically if for any $\xi_{i}(0), i \in \mathcal{I},\left\|\xi_{i}(t)-\xi_{j}(t)\right\| \rightarrow 0$ as $t \rightarrow \infty$ for each $(i, j) \in \mathcal{I}$.

\section{B. Continuous-time Consensus}

The following theorem is our main technical result.

Theorem 3.2: Given switching interaction topologies and zero transmission or communication noise, the Kalman consensus scheme given in Eqs. (1)-(3) achieves asymptotic consensus if there exist infinitely many consecutive uniformly bounded time intervals such that the union of the interaction graph across each interval has a spanning tree.

The proof of this theorem depends upon the following five lemmas.

Lemma 3.3: Let $C(t)=\left[c_{i j}(t)\right] \in M_{n}(\mathbb{R})$ be piecewise continuous, where $c_{i j} \geq 0, i \neq j$, and $\sum_{j} c_{i j}=0$. Let $\Phi_{C}\left(t, t_{0}\right)$ be the corresponding transition matrix. Then $\Phi_{C}\left(t, t_{0}\right)$ is a stochastic matrix with positive diagonal entries for any $t \geq t_{0}$.

Proof: From [18], we know that

$$
\begin{aligned}
& \Phi_{C}\left(t, t_{0}\right) \\
& =I+\int_{t_{0}}^{t} C\left(\sigma_{1}\right) d \sigma_{1}+\int_{t_{0}}^{t} C\left(\sigma_{1}\right) \int_{t_{0}}^{\sigma_{1}} C\left(\sigma_{2}\right) d \sigma_{2} d \sigma_{1}+\cdots .
\end{aligned}
$$

Noting that $C(t) \mathbf{1}=0$, where $\mathbf{1}$ is a column vector of ones, we can verify that $\Phi_{C}\left(t, t_{0}\right) \mathbf{1}=\mathbf{1}$.

Note that $C(t)$ can be written as $B(t)-\mu I_{n}$, where $B(t)$ is a nonnegative matrix and $\mu$ is a constant greater than $\max _{\tau \in\left[t_{0}, t\right]} \max _{i \in \mathcal{I}}\left|c_{i i}(\tau)\right|$. It is straightforward to see that $\frac{d}{d t} \Phi_{C}\left(t, t_{0}\right)=C(t) \Phi_{C}\left(t, t_{0}\right)$ and $\frac{d}{d t}\left[\Phi_{B}\left(t, t_{0}\right) e^{-\mu\left(t-t_{0}\right)}\right]=$ $B(t) \Phi_{B}\left(t, t_{0}\right) e^{-\mu\left(t-t_{0}\right)}-\mu \Phi_{B}\left(t, t_{0}\right) e^{-\mu\left(t-t_{0}\right)}=(B(t)-$ $\left.\mu I_{n}\right) \Phi_{B}\left(t, t_{0}\right) e^{-\mu\left(t-t_{0}\right)}=C(t) \Phi_{B}\left(t, t_{0}\right) e^{-\mu\left(t-t_{0}\right)}$, and that $\Phi_{C}\left(t_{0}, t_{0}\right)=\Phi_{B}\left(t_{0}, t_{0}\right) e^{-\mu\left(t_{0}-t_{0}\right)}=I$. Therefore, we obtain $\Phi_{C}\left(t, t_{0}\right)=\Phi_{B}\left(t, t_{0}\right) e^{-\mu\left(t-t_{0}\right)}$. From Eq. (6), it is straightforward to see that $\Phi_{B}\left(t, t_{0}\right)$ is nonnegative and has positive diagonal entries. Therefore, it follows that $\Phi_{C}\left(t, t_{0}\right)$ is nonnegative and has positive diagonal entries. Combining these arguments implies that the transition matrix $\Phi_{C}\left(t, t_{0}\right)$ is a stochastic matrix with positive diagonal entries. 
Lemma 3.4: Let $C(t)=\left[c_{i j}(t)\right] \in M_{n}(\mathbb{R})$ and $\tilde{C}=$ $\left[\tilde{c}_{i j}(t)\right] \in M_{n}(\mathbb{R})$ be continuous on $t \in[\tau, s]$, where $s>\tau$ such that $c_{i j}(t) \geq 0$ and $\tilde{c}_{i j}(t) \geq 0, \forall i \neq j$, and $\sum_{j=1}^{n} c_{i j}(t)=\sum_{j=1}^{n} \tilde{c}_{i j}(t)=0$. Let $\Phi_{C}(s, \tau)$ and $\Phi_{\tilde{C}}(s, \tau)$ be the corresponding transition matrices. Also let the graph associated with $C(t)$ be fixed for $t \in[\tau, s]$ and suppose that $\tilde{C}(t)$ corresponds to the same fixed graph as $C(t)$. Then the graph of $C(t)$ is a subset of the graph of $\Phi_{C}(s, \tau)$ and $\Phi_{C}(s, \tau) \sim \Phi_{\tilde{C}}(s, \tau)$.

Proof: Let $C(t)=B(t)-\mu I_{n}$, where $B(t)$ is a nonnegative matrix and $\mu$ is a constant greater than $\max _{t \in[\tau, s]} \max _{i \in \mathcal{I}}\left|c_{i i}(t)\right|$. Following Lemma 3.3, we know that $\Phi_{C}(s, \tau)=\Phi_{B}(s, \tau) e^{-\mu(s-\tau)}$. Note that the graphs associated with $C(t)$ and $B(t)$ are the same, so are the graphs associated with $\Phi_{C}(s, \tau)$ and $\Phi_{B}(s, \tau)$. Therefore from Eq. (6), we can see that $\Phi_{B}(s, \tau) \geq \int_{\tau}^{s} B\left(\sigma_{1}\right) d \sigma_{1}$, where $\int_{\tau}^{s} B\left(\sigma_{1}\right) d \sigma_{1} \sim B(t)$ for $t \in[\tau, s]$, or in other words, the graph associated with $B(t)$ for $t \in[\tau, s]$ is a subset of the graph associated with $\Phi_{B}(s, \tau)$. Therefore, the graph associated with $C(t)$ for $t \in[\tau, s]$ is a subset of the graph associated with $\Phi_{C}(s, \tau)$.

Note that $\Phi_{\tilde{C}}(s, \tau)=\Phi_{\tilde{B}}(s, \tau) e^{-\tilde{\mu}(s-\tau)}$, where $\tilde{C}=$ $\tilde{B}-\tilde{\mu} I_{n}$. In order to show that $\Phi_{C}$ is of the same type as $\Phi_{\tilde{C}}$, we need to show that $\Phi_{B}$ is of the same type as $\Phi_{\tilde{B}}$. Note that $B$ and $\tilde{B}$ are of the same type since they correspond to the same graph. By writing $\Phi_{B}$ and $\Phi_{\tilde{B}}$ as in Eq. (6) and comparing each term, Lemma 3.1 implies that each corresponding term is of the same type, which in turn implies that $\Phi_{B}(s, \tau)$ and $\Phi_{\tilde{B}}(\tilde{s}, \tilde{\tau})$ are of the same type.

Lemma 3.5: Let $S_{A}=\left\{A_{1}, A_{2}, \cdots, A_{\ell}\right\}$ be a set of stochastic matrices with positive diagonal entries. If the graph associated with $A_{i}$ has a spanning tree, then $A_{i}$ is SIA. If the union of the graphs of matrices $A_{i}, i=1, \cdots, \ell$, has a spanning tree, then the matrix product $\prod_{i=1}^{\ell} A_{i}$ is SIA. Proof: The first statement is shown in Corollary 3.5 and Lemma 3.7 in [8]. For the second statement, note that the product of stochastic matrices is still a stochastic matrix. Also note that $\Pi_{i=1}^{\ell} A_{i} \geq \gamma \sum_{i=1}^{\ell} A_{i}$ for some $\gamma>0$ according to Lemma 2 in [4]. Since the union of the graphs of matrices in $S_{A}$ has a spanning tree, it is obvious that the graph associate with $\sum_{i=1}^{\ell} A_{i}$ has a spanning tree. Therefore, it can be seen that the graph associated with the matrix product has a spanning tree, which in turn implies, from the first statement of the Lemma, that the matrix product is SIA.

Lemma 3.6: Let $C(t)=\left[c_{i j}(t)\right] \in M_{n}(\mathbb{R})$ be piecewise continuous for $t \in[\tau, s]$, where $s>\tau$ is bounded, $c_{i j} \geq 0$, $i \neq j$, and $\sum_{j} c_{i j}=0$. If the union of the directed graphs of matrix $C(t)$ for $t \in[\tau, s]$ has a spanning tree, then the transition matrix $\Phi_{C}(s, \tau)$ is SIA.

Proof: Note that $\Phi_{C}(s, \tau)$ = $\Phi_{C}\left(s, t_{\ell}\right) \Phi_{C}\left(t_{\ell}, t_{\ell-1}\right) \cdots \Phi_{C}\left(t_{1}, \tau\right), \quad$ where $t_{j}$, $j=1, \cdots, \ell$, denotes the times when $C(t)$ is discontinuous. From Lemma 3.4, we know that the graph associated with $C(t)$ for each $t \in\left[t_{i-1}, t_{i}\right]$ is a subset of the graph associated with $\Phi_{C}\left(t_{i}, t_{i-1}\right), i=1, \cdots, \ell+1$. In other words, if the union of the directed graphs of matrix $C(t)$ has a spanning tree, so does the union of the directed graphs of the corresponding transition matrices. Also note from Lemma 3.4 that each $\Phi_{C}\left(t_{i}, t_{i-1}\right)$ is a stochastic matrix with positive diagonal entries. The proof then follows from Lemma 3.5.

Before moving on, we need the following definition from [17]. Given a stochastic matrix $S=\left[s_{i j}\right] \in M_{n}(\mathbb{R})$, define $\lambda(S) \triangleq 1-\min _{i_{1}, i_{2}} \sum_{j} \min \left(s_{i_{1} j}, s_{i_{2} j}\right)$. Note that $\lambda(S) \leq 1$ for any stochastic matrix $S$. If $\lambda(S)<1, S$ is called a scrambling matrix. $\lambda(S)=0$ if and only if the rows of $S$ are identical. The introduction of $\lambda$ will be useful for the proof of Theorem 3.2.

Lemma 3.7: (See [17].) Let $\mathcal{S}=\left\{S_{1}, S_{2}, \cdots, S_{k}\right\}$ be a finite set of SIA matrices with the property that for each sequence $S_{i_{1}}, S_{i_{2}}, \cdots, S_{i_{j}}$ of positive length, the matrix product $S_{i_{j}} S_{i_{j}-1} \cdots S_{i_{1}}$ is SIA. Then for each infinite sequence $S_{i_{1}}, S_{i_{2}}, \cdots$ there exists a column vector $\nu$ such that

$$
\lim _{j \rightarrow \infty} S_{i_{j}} S_{i_{j-1}} \cdots S_{i_{1}}=\mathbf{1} \nu^{T} .
$$

In addition, in the case that $\mathcal{S}$ is an infinite set, $\lambda(W)<1$, where $W=S_{k_{1}} S_{k_{2}} \cdots S_{k_{N_{t}+1}}$ and $N_{t}$ is defined as the number of different types of all $n \times n$ SIA matrices. Furthermore, if there exists a constant $0 \leq d<1$ satisfying $\lambda(W) \leq d$, then Eq. (7) also holds.

Proof: See Lemma 4 and the concluding remarks in [17].

\section{Proof of Theorem 3.2:}

From Eq. (1), we see that $P_{i}$ is a uniformly lower bounded positive scalar since $Q_{i}$ is uniformly lower bounded. Also noting that $-P_{i}\left[\sum_{j} g_{i j}(t)\left(P_{j}+\Omega_{i j}\right)^{-1}\right] P_{i} \leq$ $-P_{i}^{2} /\left(P_{i}+\Omega_{i i}\right)$, we know that $P_{i}$ is uniformly upper bounded. From Eq. (2), we can see that $K_{i j}(t), \forall i \neq j$, is a uniformly lower and upper bounded positive scalar.

Let $t_{0}, t_{1}, \cdots$ be an infinite time sequence corresponding to the times at which graph $\mathcal{G}(t)$ switches topology. Since the interaction topology cannot switch infinitely fast, we assume that $t_{i}-t_{i-1} \geq t_{L}, \forall i=1,2, \cdots$. Note that each interval $\left[t_{i-1}, t_{i}\right)$ can be divided into finite or infinite number of subintervals such that the length of each subinterval is greater than or equal to $t_{L}$ but less than or equal to $t_{M}=2 t_{L}$ and the graph on each subinterval is fixed. Relabel these subintervals as $s_{0}, s_{1}, \cdots$.

Without transmission or communication noise, Eq. (3) can be rewritten in matrix form as $\dot{\xi}=\Lambda(t) \xi$, where $\xi=\left[\xi_{1}, \cdots, \xi_{n}\right]^{T}$ and $\Lambda(t)=\left[\lambda_{i j}(t)\right]$. As mentioned above, the solution can be denoted as $\xi(t)=$ $\Phi\left(t, s_{j}\right) \Phi\left(s_{j}, s_{j-1}\right) \cdots \Phi\left(s_{1}, s_{0}\right) \xi\left(s_{0}\right)$, where $\Phi$ is the transition matrix. Noting that $\lambda_{i j}(t)=g_{i j}(t) K_{i j}(t), \forall j \neq i$, and $\sum_{j} \lambda_{i j}(t)=0$, we know that $\Lambda(t)$ is continuous and satisfies the hypothesis of Lemma 3.4 for $t \in\left[s_{j-1}, s_{j}\right]$. Noting that $K_{i j}(t)$ is uniformly lower and upper bounded, we know that each nonzero, that is, positive, entry $\lambda_{i j}$, where $i \neq j$, satisfies the property that $\lambda_{i j} \in\left[\lambda_{L}, \lambda_{M}\right]$, which is a compact set. In addition, $\lambda_{i i}=-\sum_{j \neq i} \lambda_{i j}$, which is also in a compact set. In the case that the interaction topology is switching with time, there are a finite number of possible interaction topologies. For each possible interaction topology, note that matrix $\Lambda(t)$ has the same structure in the sense that positive, zero, and negative entries are in the same places for $t \in\left[s_{j-1}, s_{j}\right]$. From Lemma 3.4, each transition matrix $\Phi\left(s_{j}, s_{j-1}\right)$ is a stochastic matrix, where $t_{L} \leq s_{j}-s_{j-1} \leq t_{M}$, and $\Phi\left(s_{j}, s_{j-1}\right)$ is of constant type over this interval, for each possible interaction 
topology. Combining the above arguments with the fact that $\Phi\left(s_{j}, s_{j-1}\right)$ is a continuous function of $\lambda_{i j}(t)$ for $t \in\left[s_{j-1}, s_{j}\right]$, we see that each nonzero entry of $\Phi\left(s_{j}, s_{j-1}\right)$ is lower bounded for each possible interaction topology. It is straightforward to see that there are only finitely many types for $\Phi\left(s_{j}, s_{j-1}\right)$. We know that there exists a sequence of unions of the directed interaction graphs across some time intervals and each union is uniformly bounded and has a spanning tree. Thus the transition matrix $\Phi^{(k)}$ for each union is a product of finitely many matrices $\Phi\left(s_{k_{i}}, s_{k_{i-1}}\right)$. From Lemma 3.1, the type of $\Phi^{(k)}$ is uniquely decided by the order and type of each element in its product. Also, from Lemma 3.6, we know that each $\Phi^{(k)}$ is SIA. In addition, noting that the graph associated with each $\Phi^{(k)}$ has a spanning tree, we see that any number of products of $\Phi^{(k)}$ is also SIA according to the second part of Lemma 3.5. Noting that $\Phi^{(k)}$ can only have finitely many types, we see that for each type of $\Phi^{(k)}$ its nonzero entries are lower bounded. Let $W=\Phi^{\left(j_{1}\right)} \Phi^{\left(j_{2}\right)} \cdots \Phi^{\left(j_{N_{t}+1}\right)}$. From the second part of Lemma 3.7, we know that $\lambda(W)<1$. Note that $W$ can only have finite many types, denoted as $W_{t}$. In order to show that $\lambda(W) \leq d<1$, it is sufficient to show that for each type, there exists a $0 \leq d_{i}<1$ such that $\lambda(W) \leq d_{i}$. This can be verified by noting that the nonzero entries of $W$ are lower bounded for each type. Let $d=\max \left\{d_{1}, d_{2}, \cdots, d_{W_{t}}\right\}$. It is obvious that $\lambda(W) \leq d$. From Lemma 3.7, we can show that $\xi(t) \rightarrow \mathbf{1} \nu^{T} \xi(0)$, where $\nu$ is a nonnegative column vector.

In previous results on consensus [4], [8], the coefficient matrix $C(t)$ was assumed to be piecewise constant with finite dwell time, and elements drawn from a finite set. The following corollary of Theorem 3.2 shows that these conditions can be relaxed.

Corollary 3.8: Let $\dot{\xi}=C(t) \xi$, where $C(t)=\left[c_{i j}(t)\right] \in$ $M_{n}(\mathbb{R})$ is piecewise continuous, $c_{i j} \geq 0, i \neq j, \sum_{j} c_{i j}=0$, and each nonzero entry $c_{i j}, i \neq j$, is both uniformly lower and upper bounded. Under switching interaction topologies, $\xi_{i}$ achieves consensus if there exist infinite many consecutive uniformly bounded time intervals such that the union of the interaction graph across each such interval has a spanning tree.

\section{Discrete-time Consensus}

Theorem 3.9: Given switching interaction topologies and zero transmission or communication noise, the discrete-time Kalman consensus scheme listed in Eq. (4)-(5) achieves asymptotic consensus if there exist infinitely many consecutive uniformly bounded time intervals such that the union of the interaction graph across each interval has a spanning tree.

Proof: Without transmission or communication noise, Eq. (5) can be written as

$$
\begin{aligned}
\xi_{i}[k+1] & =\left[1-P_{i}[k+1] \sum_{j \neq i} g_{i j}[k]\left(P_{j}[k]+\Omega_{i j}\right)^{-1}\right] \xi_{i}[k] \\
& +P_{i}[k+1] \sum_{j \neq i}\left[g_{i j}[k]\left(P_{j}[k]+\Omega_{i j}\right)^{-1} \xi_{j}[k]\right] .
\end{aligned}
$$

Note that each weighting factor of $\xi_{\ell}$ is less than or equal to 1 and the sum of the weighting factors of $\xi_{\ell}$ is equal to 1 , where $\ell \in \mathcal{I}$. Letting $\xi=\left[\xi_{1}, \cdots, \xi_{n}\right]^{T}$, we can rewrite Eq. (8) as $\xi[k+1]=D[k] \xi[k]$, where it can be verified that
$D[k]$ is a stochastic matrix with positive diagonal entries. In addition, for each possible interaction topology, $D[k]$ is of the same type and its nonzero entries are lower bounded.

We know that there exists a sequence of unions of the directed interaction graphs across some time intervals and each union is uniformly bounded and has a spanning tree. Let $D^{(i)}$ be the product of matrices $D[k]$ over the $i^{\text {th }}$ union. Note that each $D^{(i)}$ is SIA from Lemma 3.5. As a result, the proof follows the same reasoning as the proof of Theorem 3.2 with $D^{(i)}$ playing the role of $\Phi^{(k)}$.

\section{Conclusion}

This paper has considered the problem of consensus seeking with relative uncertainty in distributed multi-agent systems. We have proposed discrete-time and continuoustime Kalman filter-like consensus schemes that are appropriate when different agents in the group may have different confidences about their information state. Sufficient conditions have been shown for consensus seeking using the proposed consensus schemes under switching interaction topologies.

\section{REFERENCES}

[1] W. Ren, R. W. Beard, and T. W. McLain, "Coordination variables and consensus building in multiple vehicle systems," in Cooperative Control (V. Kumar, N. E. Leonard, and A. S. Morse, eds.), vol. 309, pp. 171-188, Springer-Verlag Series: Lecture Notes in Control and Information Sciences, 2004.

[2] T. W. McLain and R. W. Beard, "Coordination variables, coordination functions, and cooperative timing missions," AIAA Journal of Guidance, Control, and Dynamics, vol. 28, pp. 150-161, January 2005.

[3] J. A. Fax and R. M. Murray, "Information flow and cooperative control of vehicle formations," IEEE Transactions on Automatic Control, vol. 49, pp. 1465-1476, September 2004.

[4] A. Jadbabaie, J. Lin, and A. S. Morse, "Coordination of groups of mobile autonomous agents using nearest neighbor rules," IEEE Transactions on Automatic Control, vol. 48, pp. 988-1001, June 2003.

[5] R. Olfati-Saber and R. M. Murray, "Consensus problems in networks of agents with switching topology and time-delays," IEEE Transactions on Automatic Control, vol. 49, pp. 1520-1533, September 2004.

[6] L. Moreau, "Stability of multi-agent systems with time-dependent communication links," IEEE Transactions on Automatic Control, vol. 50, pp. 169-182, February 2005.

[7] Z. Lin, M. Broucke, and B. Francis, "Local control strategies for groups of mobile autonomous agents," IEEE Transactions on Automatic Control, vol. 49, no. 4, pp. 622-629, 2004.

[8] W. Ren and R. W. Beard, "Consensus seeking in multi-agent systems under dynamically changing interaction topologies," IEEE Transactions on Automatic Control, May 2005.

[9] L. Xiao and S. Boyd, "Fast linear iterations for distributed averaging," Systems and Control Letters, vol. 53, pp. 65-78, 2004.

[10] B. Barshan and H. F. Durrant-Whyte, "Inertial navigation systems for mobile robots," IEEE Transactions on Robotics and Automation, vol. 11, pp. 328-342, June 1995.

[11] M. E. Cannon, "Integrated GPS-INS for high-accuracy road positioning," Journal of Surveying Engineering, vol. 118, pp. 103-117, November 1992.

[12] S. Ronnback, "Development of an INS/GPS navigation loop for an UAV," Master's thesis, Luleå University of Technology, 2000.

[13] A. H. Jazwinski, Stochastic Processes and Filtering Theory, vol. 64 of Mathematics in Science and Engineering. New York, New York: Academic Press, Inc., 1970.

[14] F. L. Lewis, Optimal Estimation: With an Introduction to Stochastic Control Theory. New York, New York: John Wiley \& Sons, 1986.

[15] C. Godsil and G. Royle, Algebraic Graph Theory. New York: Springer Graduate Texts in Mathematics \#207, 2001.

[16] R. A. Horn and C. R. Johnson, Matrix Analysis. Cambridge University Press, 1985.

[17] J. Wolfowitz, "Products of indecomposable, aperiodic, stochastic matrices," Proceedings of the American Mathematical Society, vol. 15, pp. 733-736, 1963

[18] W. J. Rugh, Linear System Theory. Englewood Cliffs, New Jersey: Prentice Hall, 2nd ed., 1996. 